\title{
Comparative analysis of the gait disorder of normal pressure hydrocephalus and Parkinson's disease
}

\author{
H Stolze, J P Kuhtz-Buschbeck, H Drücke, K Jöhnk, M Illert, G Deuschl
}

\begin{abstract}
Objectives-Comparative gait analyses in neurological diseases interfering with locomotion are of particular interest, as many hypokinetic gait disorders have the same main features. The aim of the present study was (1) to compare the gait disturbance in normal pressure hydrocephalus and Parkinson's disease; (2) to evaluate which variables of the disturbed gait pattern respond to specific treatment in both diseases; and (3) to assess the responsiveness to visual and acoustic cues for gait improvement.
\end{abstract}

Methods-In study 1 gait analysis was carried out on 11 patients with normal pressure hydrocephalus, 10 patients with Parkinson's disease, and 12 age matched healthy control subjects, on a walkway and on a treadmill. In study 2, patients with normal pressure hydrocephalus were reinvestigated after removal of $30 \mathrm{ml} \mathrm{CSF}$, and patients with Parkinson's disease after administration of $150 \mathrm{mg}$ levodopa. In part 3 visual cues were provided as stripes fixed on the walkway and acoustic cues as beats of a metronome.

Results-The gait disorder in both diseases shared the feature of a reduced gait velocity, due to a diminished and highly variable stride length. Specific features of the gait disturbance in normal pressure hydrocephalus were a broad based gait pattern with outward rotated feet and a diminished height of the steps. After treatment in both diseases, the speed increased, due to an enlarged stride length, now presenting a lower variability. All other gait variables remained unaffected. External cues only mildly improved gait in normal pressure hydrocephalus, whereas they were highly effective in raising the stride length and cadence in Parkinson's disease.

Conclusion-The gait pattern in normal pressure hydrocephalus is clearly distinguishable from the gait of Parkinson's disease. As well as the basal ganglia output connections, other pathways and structures most likely in the frontal lobes are responsible for the gait pattern and especially the disturbed dynamic equilibrium in normal pressure hydrocephalus. Hypokinesia and its responsiveness to external cues in both diseases are assumed to be an expression of a disturbed motor planning. (f Neurol Neurosurg Psychiatry 2001;70:289-297)

Keywords: gait analysis; Parkinson's disease; normal pressure hydrocephalus
Hypokinetic gait disorders occur in many different neurological diseases, such as Parkinson's disease, normal pressure hydrocephalus, subcortical arteriosclerotic encephalopathy (Binswanger's disease), Alzheimer's disease, and other rare disorders such as progressive supranuclear palsy and corticobasal ganglionic degeneration. It has been shown that about $35 \%$ of the gait disorders in a neurological referral practice are hypokinetic. ${ }^{1}$ Of these, about $55 \%$ are related to Parkinson's disease and normal pressure hydrocephalus. The differential diagnosis of hypokinetic gait disorders is still unsolved clinically, because direct comparative studies of the gait pattern in the different diseases with hypokinetic gait disorders are rare. ${ }^{2-4}$

The syndrome of normal pressure hydrocephalus, first described by Adams et $a l,{ }^{5}$ is characterised by the clinical triad of gait disturbance, dementia, and urinary incontinence. Disturbance of gait is usually the first sign and considered the most important symptom..$^{6-8}$ Besides a diminished gait velocity, mostly due to a reduced stride length, the pattern is characterised by a reduced foot to floor clearance (step height) during the swing phase of the gait cycle and enlarged balance related gait measurements such as step width and foot rotation angles. ${ }^{9}$ Gait associated movements such as arm swing are unimpaired ${ }^{10}$ cocontractions of proximal muscles have been proposed to be responsible for the reduced stride length. ${ }^{10}$ After shunting or tapping of CSF the gait velocity increases by about $20 \% .^{911}$

The gait pattern in Parkinson's disease has been well described and the gait variables have been analysed quantitatively. ${ }^{12-19}$ Some measurements, such as stride length and gait velocity, are levodopa sensitive; others are levodopa resistant. ${ }^{17}$ Morris et $a l^{20-22}$ showed that the slow and shuffling gait in Parkinson's disease depends on an inability to generate an appropriate stride length, whereas the cadence is normal or increased to reach a normal gait velocity. When patients on levodopa were instructed to use acoustic or visual external cues, a normal cadence and step length were achieved. It has been proposed that these external cues may compensate for the lack of internal cues from the basal ganglia to supplementary motor cortex and primary motor cortex via the thalamus. ${ }^{20} \mathrm{~A}$ second hypothesis suggested a disturbed cortical motor set as the origin of the hypokinetic gait pattern in Parkinson's disease. ${ }^{21}$

Although the gait pattern in both diseases displays similarities including short steps, leg 
Demographic and anthropometric data of patients and controls

\begin{tabular}{llllll}
\hline Group & $n$ & Men/women & Age $(y)$ & Height $(\mathrm{cm})$ & Weight (kg) \\
\hline HC & 12 & $5 / 7$ & $74.6(5.9)$ & $166.1(7.8)$ & $66.7(9.7)$ \\
NPH & 11 & $7 / 4$ & $76.0(6.0)$ & $166.7(10.4)$ & $75.1(11.2)$ \\
PD & 10 & $6 / 4$ & $66.4(6.7)$ & $173.3(6.5)$ & $72.4(14.4)$ \\
\hline
\end{tabular}

Values are means (SD). PD=Parkinson's disease; $\mathrm{NPH}=$ normal pressure hydrocephalus; $\mathrm{HC}=$ healthy control. criteria. ${ }^{28}$ The mean disease duration was 7.7 (SD 4.8) years. The disease was diagnosed at mean age 59.5 (SD 9.5) years. During on levodopa the mean score in the unified Parkinson's disease rating scale (part III) was 29.6 (SD 16.0) points.

\section{Healthy controls}

Healthy subjects were included when a neurological examination was normal and they were free of any signs of a disease interfering with unrestrained gait. The group was age and sex matched to the patients with normal pressure hydrocephalus. pressure hydrocephalus to improve the gait, as in Parkinson's disease, has so far not been investigated.

It was the aim of the present study to compare the gait pattern in normal pressure hydrocephalus and Parkinson's disease to find diagnostic signs for their differential diagnosis. In both diseases we have further studied the effect of therapeutic interventions. These data give further information on the pathophysiology underlying hypokinetic gait disorders.

\section{Methods}

SUBJECTS

Eleven patients with idiopathic normal pressure hydrocephalus and 10 patients with idiopathic Parkinson's disease (Hoehn and Yahr stage 2.7 (SD 0.4) were included in the study. All were inpatients of the department of neurology. Twelve healthy subjects from senior clubs of the community served as controls (see table for demographic data of the patients and control subjects). All patients and controls gave their informed consent to participate in the study, which was approved by the local ethics committee.

\section{INCLUSION CRITERIA}

Normal pressure hydrocephalus

Patients were included in the study when they fulfilled the clinical criteria for idiopathic normal pressure hydrocephalus. ${ }^{24}$ A standardised cranial MRI (Siemens Magnetom Expert) was performed before CSF tapping, and the Evans ratio (ratio of the greatest distance of the lateral walls in the frontal horns of the first and second ventricles and diameter of the inner table of the skull, both in the transverse plane) was calculated..$^{25}$ For diagnosis of normal pressure hydrocephalus, the cranial MRI had to disclose an enlarged ventricular system (Evans ratio $>0.31$; see Soelberg Sorensen et $a l^{26}$ ) without a moderate or severe cortical atrophy. ${ }^{25}$ Microvascular lesions of the white matter were accepted only to a mild degree. All patients had a moderate to severe gait disorder. They had disturbed cognitive functions and urinary incontinence to a varying degree. For inclusion the intrathecal pressure measured in a lying position during CSF tapping had to be below $20 \mathrm{~cm} \mathrm{H}_{2} \mathrm{O}$. Patients presenting with additional neurological or orthopaedic disorders interfering with an unrestrained gait pattern were excluded.

Parkinson's disease

Idiopathic Parkinson's disease was diagnosed according to the United Kingdom brain bank
EXPERIMENTAL PROTOCOL (STUDIES 1 AND 2) In study 1 the pathology of the gait pattern was studied in the two untreated patient groupsthat is, before CSF tapping in normal pressure hydrocephalus and before levodopa treatment in Parkinson's disease. Study 2 was designed to analyse the effect of therapeutically interventions on the gait variables. In normal pressure hydrocephalus locomotion analysis of patients was carried out 24 hours after tapping of $30 \mathrm{ml}$ CSF, in patients with Parkinson's disease 1 hour after oral administration of $150 \mathrm{mg}$ levodopa in a fast release preparation. In the controls, gait analysis was performed once.

In these experiments we measured the following spatiotemporal gait variables during overground locomotion: gait velocity, stride length, cadence, step width, foot angle (outward rotation denoted as positive), gait cycle, stance, swing, and double limb support phase duration. During treadmill locomotion a further seven variables were recorded, mainly describing the kinematics of the gait (maximum and minimum angular excursions of the hip, knee, and ankle joint and the step height). Each experiment started with four trials of overground gait analysis (corresponding to 15-20 gait cycles), in which the patients were instructed to walk at their preferred "natural" gait velocity. The walking speed was calculated as the mean velocity of four walkway trials. For treadmill locomotion the treadmill speed was adjusted to $0.28 \mathrm{~m} / \mathrm{s}$ $(1.0 \mathrm{~km} / \mathrm{h})$. All subjects were able to walk safely at this speed). The subjects were allowed to use the handrails of the treadmill for support; if necessary they were additionally secured with a safety belt suspended from the ceiling of the laboratory (no weight support). Before recording the subjects were given 5 minutes to familiarise themselves with treadmill locomotion. Three trials of 20 seconds in duration were recorded. From these trials, 15-20 consecutive walking cycles were averaged for off line analysis and calculation of the different gait variables. The retest reliability of these gait variables has been previously demonstrated. ${ }^{29}$

EXPERIMENTAL PROTOCOL (STUDY 3)

Study 3 investigated the use of external cues to improve the gait pattern. During overground locomotion each cue condition was tested in four trials before and after treatment (CSF tapping and levodopa administration). To increase the cadence the patients were instructed to pace their step frequency to the beat 
of an electronic metronome, which was adjusted to the step frequency of the controls (110steps/min). To improve the diminished step length the patients were asked to step on black stripes (width $5 \mathrm{~cm}$ ) which were placed at a distance of $60 \mathrm{~cm}$ on the walkway, the distance representing the step length of the controls. From these trials the gait velocity, the cadence, and the step length were derived. To compare the results of the two patient groups with the controls, the $95 \%$ confidence interval ( $95 \% \mathrm{CI})$ of the mean values were calculated and compared with the means of the three gait variables in the cue task.

METHODS FOR GAIT ANALYSIS

The temporospatial gait measurements (except step height) were investigated during free speed locomotion on a walkway. The walkway consisted of a long soft carpet (length $13 \mathrm{~m}$, width 1 $\mathrm{m})$, rolled on a well illuminated laboratory floor. A transparent draft paper covered the central part $(5 \mathrm{~m})$ of the walkway. Two small brass plates, prepared with punched out peaks, not noticeable to the subjects during walking, were fixed under the sole of each of the usual walking shoes of the subjects. The punched out peaks imprinted the footmarks on the draft paper. For evaluation of the spatial measurements, the imprinting of the footmarks were measured using an engineer's drawing board. Gait velocity was calculated by measuring the time each subject needed to cover the distance of 5 metres, using infrared light barriers. These time measurements were also used to calculate the cadence. To separate the different phases of the walking cycle the gait was filmed with a video camera (50 frames/s, high speed shutter $0.01 \mathrm{~s}$ ). The videotapes were analysed using self developed software. Detailed descriptions of the method used for the analysis of overground locomotion are given elsewhere. ${ }^{30} 31$

The kinematics of the limb movements and the step height were measured on a belt driven treadmill (Jaeger-Toennies). During treadmill locomotion the subjects wore their normal walking shoes. The gait was recorded with an infrared movement analysis system (Qualisys, Sandvälen, Sweden), consisting of four infrared cameras and video processors $(50 \mathrm{~Hz}$ sampling rate) connected to a Macintosh computer. Seven infrared light reflective spherical markers were attached to each leg. The hip joint angle was measured between the markers fixed over the anterior superior iliac spine, the trochanter, and the thigh. Unfortunately, the markers over the iliac spine and the trochanter were often obscured by the swinging arms or the hand rails, which meant that the hip joint excursions were evaluable in all patients. The knee joint angle was determined between the markers attached to the thigh, the knee, and the lateral malleolus. The excursion of the ankle joint was calculated as the angle between the knee, lateral malleolus, and forefoot markers. The maximum elevation of the calcaneal marker during the swing phase of gait was defined as step height. The gait variables were calculated off line with self developed software.
STATISTICAL ANALYSIS

Means (SD) were calculated for the gait variables derived from 15-20 gait cycles in each subject during both overground and treadmill locomotion. Additionally, the coefficients of variation (CV) were calculated for stride length, step width, and foot angle to quantify the within subject variability. Differences between the gait variables recorded before and after CSF tapping in normal pressure hydrocephalus and levodopa administration in Parkinson's disease were analysed with paired $t$ tests (two tailed). The student $t$ test was also used for analysing the effects of external cues on gait before and after therapeutic interventions. Differences in the various gait variables between the three groups were compared by analysis of variance (ANOVA) for repeated measurements and Bonferroni correction. A discriminant analysis (direct method) was used to extract distinguishable gait variables in normal pressure hydrocephalus and Parkinson's disease.

\section{Results}

DIFFERENCES IN GAIT VARIABLES BETWEEN PATIENTS WITH NORMAL PRESSURE HYDROCEPHALUS, PATIENTS WITH PARKINSON'S DISEASE, AND CONTROLS (STUDY 1)

The demographic data of the patient groups and the controls are listed in the table. Figures 1 and 2 summarise the differences between the patient groups and the controls. The natural walking velocity was reduced in the patients when compared with the controls (1.18 (SD $0.22) \mathrm{m} / \mathrm{s}$ ), severely in normal pressure hydrocephalus $(0.45(\mathrm{SD} 0.18) \mathrm{m} / \mathrm{s})$, moderately in Parkinson's disease $(0.76(\mathrm{SD} 0.29) \mathrm{m} / \mathrm{s})$. In both patient groups this reduction was mainly due to a shorter stride length than to a diminished cadence. The CV of the stride length was significantly higher in the patients (normal pressure hydrocephalus 14.3 (SD 9.6)\%; Parkinson's disease 13.0 (SD 12.2)\%) than in controls (3.3 (SD 1.1)\%). The symmetry of the step length was not significantly different between the groups, although it was more asymmetric in normal pressure hydrocephalus. A freezing phenomenon was seen in three patients with normal pressure hydrocephalus and four patients with Parkinson's disease.

The duration of the phases of the walking cycle in the patients behaved according to the reduced gait velocity and the cadence (fig $1 \mathrm{~B}$ ). The equilibrium related gait variables - that is, the step width and the foot rotation angleswere characteristically altered in normal pressure hydrocephalus (fig 2), but less affected in Parkinson's disease. In normal pressure hydrocephalus the step width was significantly enlarged. Its variability, however, was reduced (19.2 (13.4)\%), when compared with the controls (59.3 (SD 28\%)) and with Parkinson's disease (41.8 (SD 24.3)\%). Further, the patients with normal pressure hydrocephalus walked with a more pronounced outward rotation of the feet than the other two groups. The CV of the foot angles was significantly lower in normal pressure hydrocephalus (24.6 (SD $15.0) \%$ ) than in the controls (36.2 (SD 21.0)\%) 
A
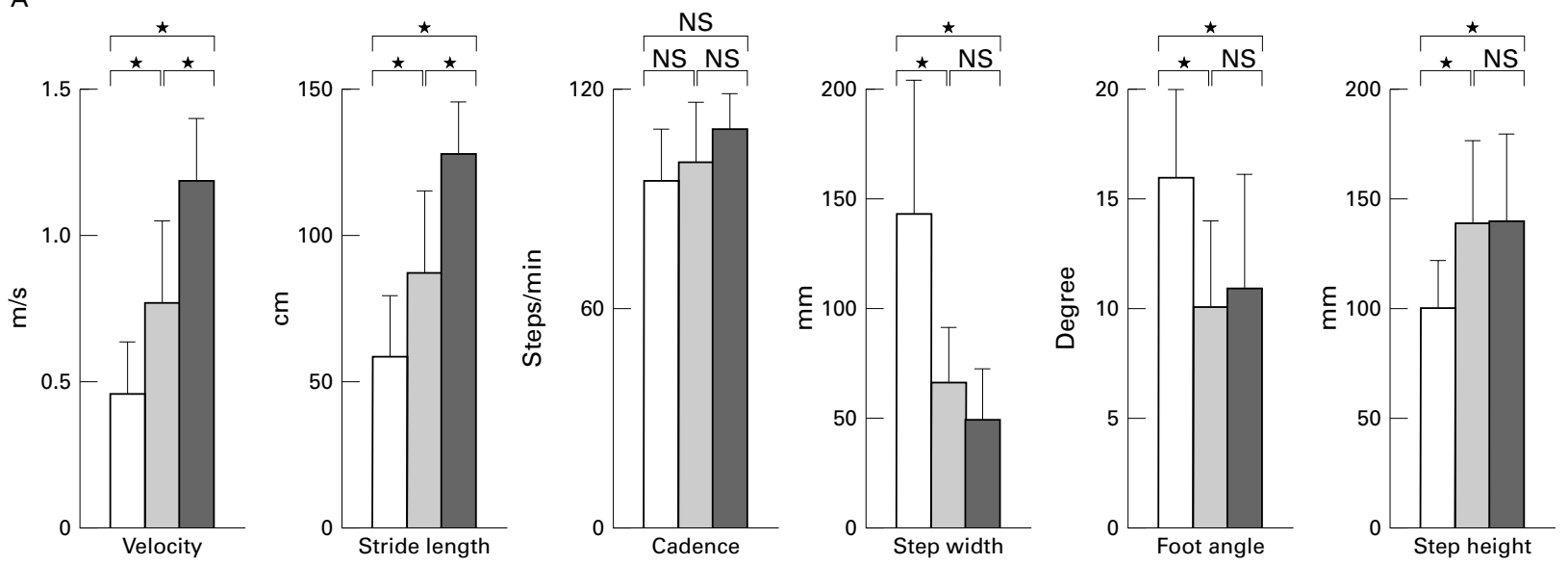

B

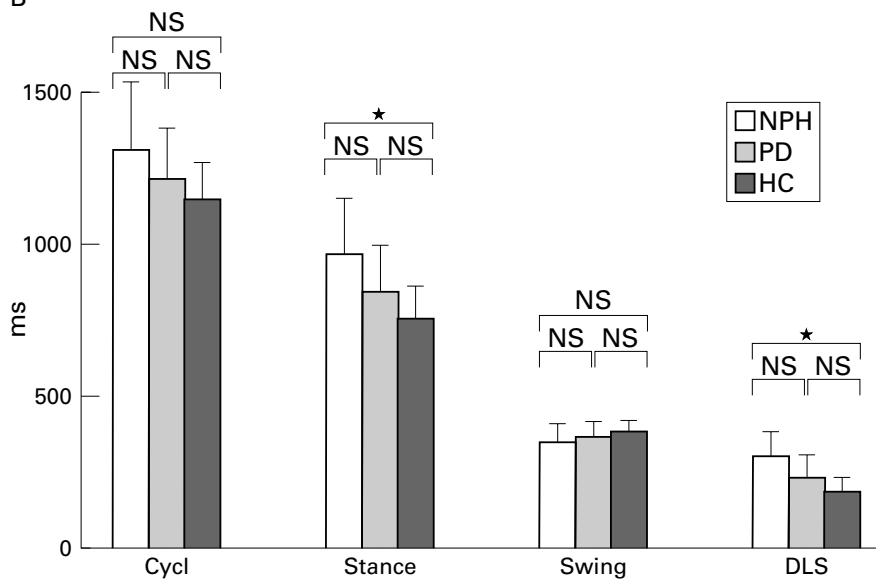

Figure 1 Differences in gait variables between patients with normal pressure hydrocephalus (NPH), Parkinson's disease (PD), and healthy controls (HC). (A) Mean (SD) of spatial gait variables and (B) temporal gait variables. Cycl=gait cycle time; Stance=stance phase duration; Swing=swing phase duration; $D L S=$ duration of the double limb support phase. The data were recorded before therapy (CSF tapping in normal pressure hydrocephalus and levodopa application in Parkinson's disease). ${ }^{\star} p<0.05 . N S=$ non-significant.

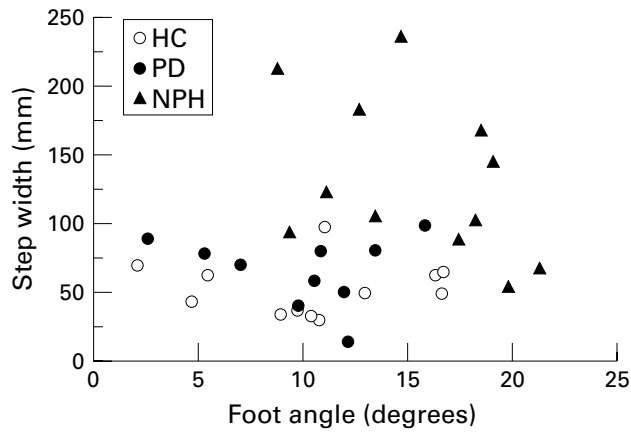

Figure 2 Step width (ordinate) plotted against the foot rotation angles (abscissa) for healthy controls (HC; full circles), Parkinson's disease (PD; open circles), and normal pressure hydrocephalus (NPH; full triangles). Note the marked differences in both variables between healthy controls and Parkinson's disease with normal pressure hydrocephalus. The regression analysis disclosed that in normal pressure hydrocephalus either the step width or the foot angles were altered, although this finding was not significant $(p=0.16)$

and Parkinson's disease (49.3 (SD 26.7)\%). The step width and the foot angles were enlarged in normal pressure hydrocephalus (fig 2) and there was a non-significant trend for imbalance to be either compensated by a larger step width or enlarged foot angles. In the discriminant analysis between the groups the step width and the foot angles were the most powerful variables in distinguishing between normal pressure hydrocephalus and Parkinson's disease (fig 2). Based on these two variables $90.48 \%$ of the patients

were correctly classified (Wilk's lambda $0.3 ; \chi^{2}$ $19.9 ; \mathrm{p}<0.001)$. If the step height was additionally included in the dicriminant analysis, $91.67 \%$ of the patients were correctly classified.

The kinematic recordings during treadmill locomotion showed a severe reduction in the angular excursions in normal pressure hydrocephalus. Thus the hip joint excursion was reduced to $9^{\circ}$, with the hip held in a more erect position, compared with the controls $\left(17^{\circ}\right)$. At the knee joint the range of motion was clearly diminished in normal pressure hydrocephalus $\left(26^{\circ} v 38^{\circ}\right.$ in controls) and a fully normal knee extension was not achieved. The kinematic tracings further disclosed that movements in the ankle joint were restricted in normal pressure hydrocephalus (range of motion $13^{\circ}$ ). Patients were not able to lift their feet to a normal height and to perform a normal dorsal extension of the forefoot during the terminal swing phase, and thus the foot struck the ground flat and not with the heel as in controls, whereas the dorsal extension of the foot in these patients was nearly normal. They were not able to lift their feet to a normal height during the swing phase, and thus the foot struck the ground flat and not with the heel as in controls. In most patients with normal pressure hydrocephalus, associated movements of the trunk and upper limbs, in particular arm swing during overground walking, were only 
slightly impaired, although this has not been evaluated quantitatively.

In Parkinson's disease the hip joint excursions were slightly reduced $\left(14^{\circ}\right)$ compared with the controls $\left(17^{\circ}\right)$. At the knee, the range of motion was nearly normal $\left(36^{\circ} v 38^{\circ}\right.$ in controls), although, as in normal pressure hydrocephalus, the knee extension was insufficient. The dorsal extension of the foot in patients with Parkinson's disease (maximum ankle joint angle $100^{\circ}$ ) was less than in controls $\left(110^{\circ}\right)$, which could be explained by a flexed and stiffened posture of the trunk throughout the gait cycle on the treadmill. Interestingly, the step height was normal in these patients. In summary, the range of motion at the joints of the lower body was more diminished in normal pressure hydrocephalus than in Parkinson's disease.

One of the distinct features in the gait patterns involved the balance related gait variables (step width and foot angles), which were enlarged in normal pressure hydrocephalus. To assess if this was a disease related feature or due to the reduced gait velocity in normal pressure hydrocephalus we investigated three further patients with Parkinson's disease off levodopa (mean age 72.7 (SD 11.5) years, Hoehn and Yahr stage III-IV). In overground condition, the patients were instructed to walk at three different speeds (at the preferred velocity, faster, slower). In all three conditions the step width (about $80 \mathrm{~mm}$ ) and the foot rotation angles (about $8^{\circ}$ ) were the same and below the values measured in patients with normal pressure hydrocephalus.

GAIT VARIABLES IN PATIENTS WITH NORMAL PRESSURE HYDROCEPHALUS AND PARKINSON'S DISEASE AFTER THERAPEUTIC INTERVENTIONS (STUDY 2)

The differences in the gait variables in normal pressure hydrocephalus before and after CSF tapping and in Parkinson's disease off and on levodopa are shown in fig $3 \mathrm{~A}$ and B. In patients with normal pressure hydrocephalus some gait variables improved after tapping of $30 \mathrm{ml} \mathrm{CSF}$. This happened in all patients, although to a different degree. The gait velocity increased by about $21 \%$. This was caused by an enlarged stride length, whereas the cadence remained unaffected. The $\mathrm{CV}$ of the stride length decreased significantly. Within the walking cycle the duration of the stance phase shortened after tapping, that of the swing phase increased, with the consequence of a shorter double limb support time (fig $3 \mathrm{~B}$ ). In the balance related gait variables and the step height no significant differences were found before and after CSF tapping. The joint angle excursions generally increased after tapping, whereas the variability decreased. The angular excursions of the hip joint increased from $9^{\circ}$ to $17^{\circ}$ (seen in six patients in whom the markers were not obscured). The knee angle excursion increased as well (from $26^{\circ}$ to $31^{\circ}$, nonsignificant), whereas no change of the angular
A

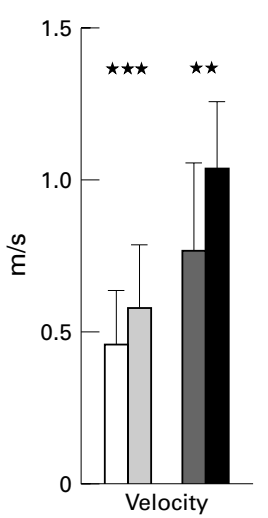

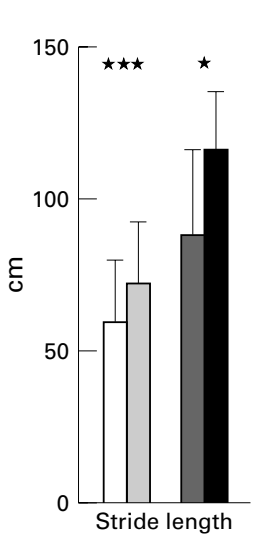
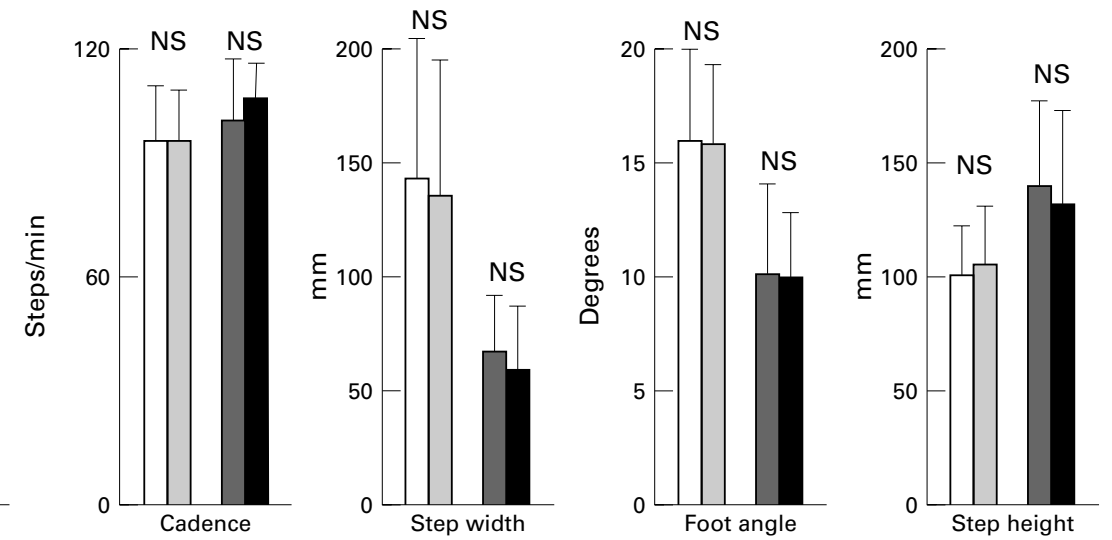

B

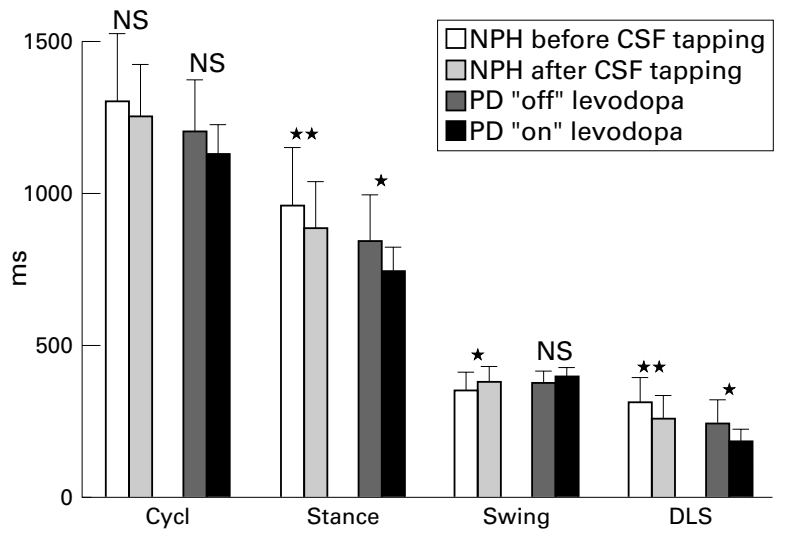

Figure 3 Differences in gait variables before and after CSF tapping in normal pressure hydrocephalus (NPH), and levodopa application in Parkinson's disease (PD). (A) Means (SD) of spatial gait variables and (B) temporal gait variables. $H C=$ healthy controls; $C y c l=$ gait cycle time; Stance=stance phase duration; Swing=swing phase duration; $D L S=$ duration of the double limb support phase. ${ }^{\star} p<0.05 ;{ }^{\star \star} p<0.01$; $* * *<0.001$. NS $=$ non-significant. 
A

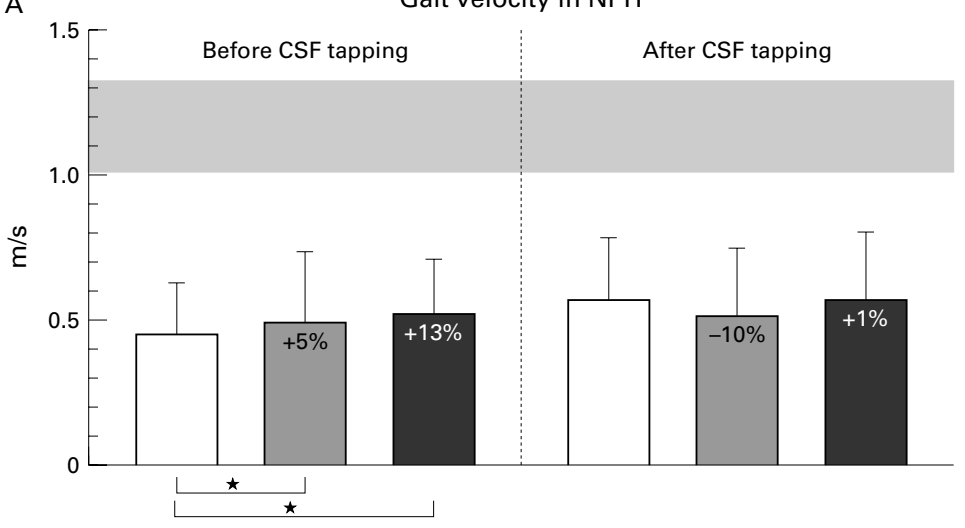

B

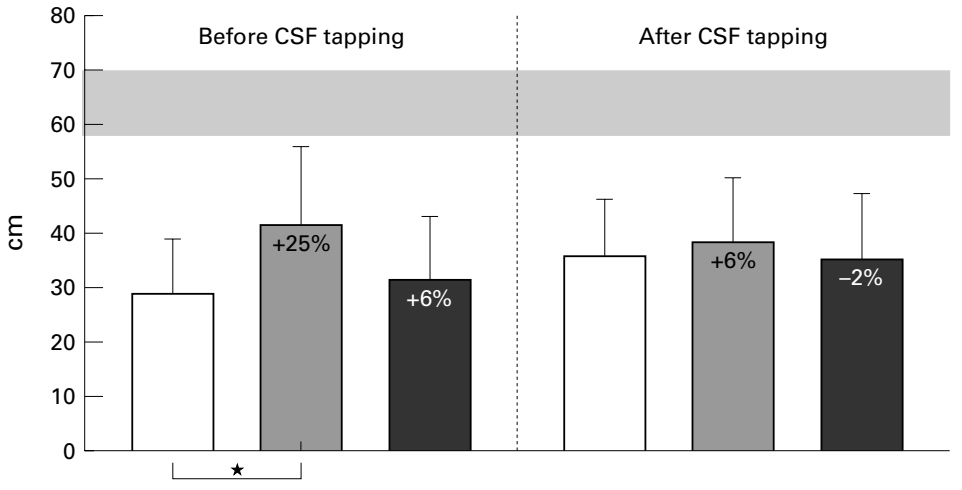

C Before CSF tapping Cadence in NPH After CSF tapping

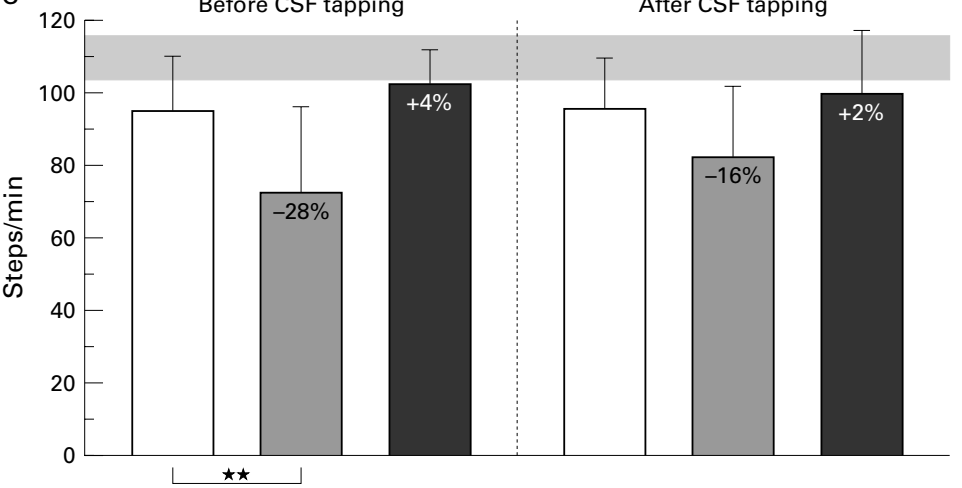

Figure 4 Effects of visual (stripes on the floor, displaying a normal step length) and acoustic cues (beat of a metronome corresponding to a normal cadence) on $(A)$ the gait velocity, (B) step length, and (C) cadence in patients with normal pressure hydrocephalus (NPH) before (left bars) and after CSF tapping (right bars). Depicted are means (SD) of the gait parameters. The horizontal grey bar depicts the $95 \%$ CI of the mean values of each gait variable in the control group. Note the diminished cadence in normal pressure

hydrocephalus, when visual cues were applied to the patients. Significant differences between the conditions without and with cues are indicated. ${ }^{\star} p<0.05 ;{ }^{\star \star} p<0.01 ;{ }^{\star \star \star} p<0.001$. (before and after CSF tapping)

excursion was seen in the ankle joint $\left(13^{\circ}\right.$ before and after tapping).

In Parkinson's disease a considerable improvement of gait was found after administration of levodopa. The gait velocity increased by about $26 \%$ due to an increase of the stride length by $24 \%$ and to an augmentation of the cadence by $5 \%$. The changes of the phases of the

walking cycle altered accordingly (shorter stance phase, shorter double limb support time, fig 3 B). The CV of the stride length decreased significantly, from $13 \%$ to $6 \%$. The step width, the foot rotation angles, and the height of the steps were not significantly altered. The angular excursion of the hip joint was not affected (from $14^{\circ}$ to $13^{\circ}$ ), but the maximum hip angle increased massively by $32^{\circ}$ (found in four patients in whom the joint excursions could be evaluated). This led to a more erect posture of the patients after levodopa administration. In the knee and ankle joints the angular excursions were not affected by levodopa

EFFECT OF EXTERNAL CUES ON THE GAIT PATTERN IN NORMAL PRESSURE HYDROCEPHALUS AND PARKINSON'S DISEASE (STUDY 3)

When patients with normal pressure hydrocephalus were instructed to increase their stride length by stepping on the stripes and walking to the beat of a metronome, the influence on the gait velocity (fig 4 A) was only mild. After CSF tapping the situation did not change and furthermore in the stripes task the gait velocity decreased. The $95 \%$ CI of the gait velocity in controls was never reached.

Visual cues (fig $4 \mathrm{~B}$ ) were effective in enlarging the step length by about $25 \%$. Pacing by a metronome enlarged the step length by $6 \%$. After CSF tapping the spontaneous step length increased, as already described; additional application of visual cues improved the step length to a minor extent and metronome walking had no effect on the stride size.

Before CSF tapping the cadence was only slightly less than in controls (fig $4 \mathrm{C}$ ). The metronome was helpful in establishing a nearly normal step frequency ( $4 \%$ increase). In the stripes task, the cadence decreased to 72 steps/ min. After therapy this constellation of effects remained more or less unchanged.

As expected, the patients with Parkinson's disease responded favourably to external cues by improving gait. When stepping on the stripes the speed of walking (fig $5 \mathrm{~A}$ ) increased by $10 \%$ and about $8 \%$ when walking to the metronome (off levodopa). After the administration of levodopa the gait velocity reached normal values, irrespective of the application of visual and acoustic cues, which had no additional effect.

Visual cues were highly effective in enlarging the step length (fig $5 \mathrm{~B}$ ) to reach normal values, when off levodopa. Again, when the patients were on levodopa, the beneficial effect of the cues was mostly saturated by the drug.

As described before, the cadence (fig $5 \mathrm{C}$ ) was not significantly lower in Parkinson's disease than in controls. When walking to the metronome off levodopa, there was a further beneficial effect on the cadence ( $4 \%$ increase to 104 steps/min). Walking on the stripes, interestingly decreased the cadence by about 15 steps/min (18\%). After levodopa administration, the cadence reached normal values in all conditions and, which is of interest, was also normal in the stripes task. 
A

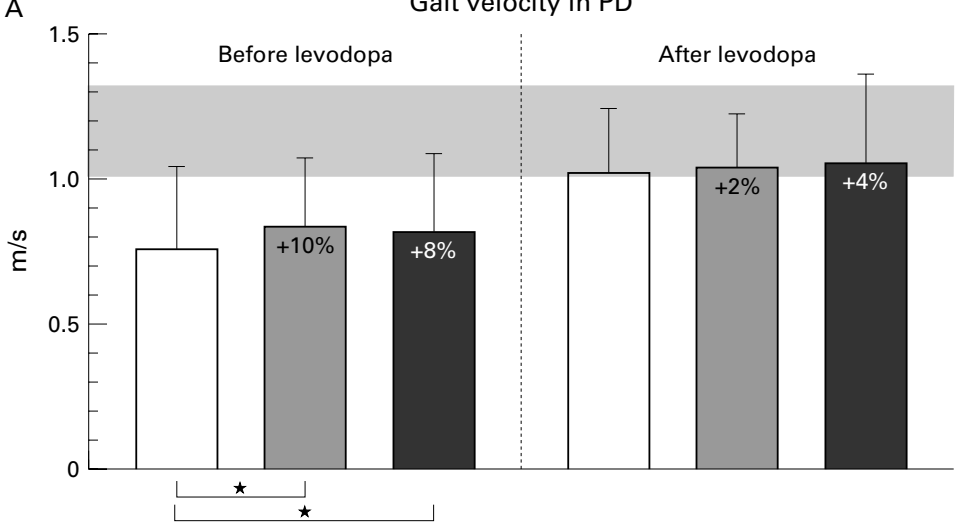

B
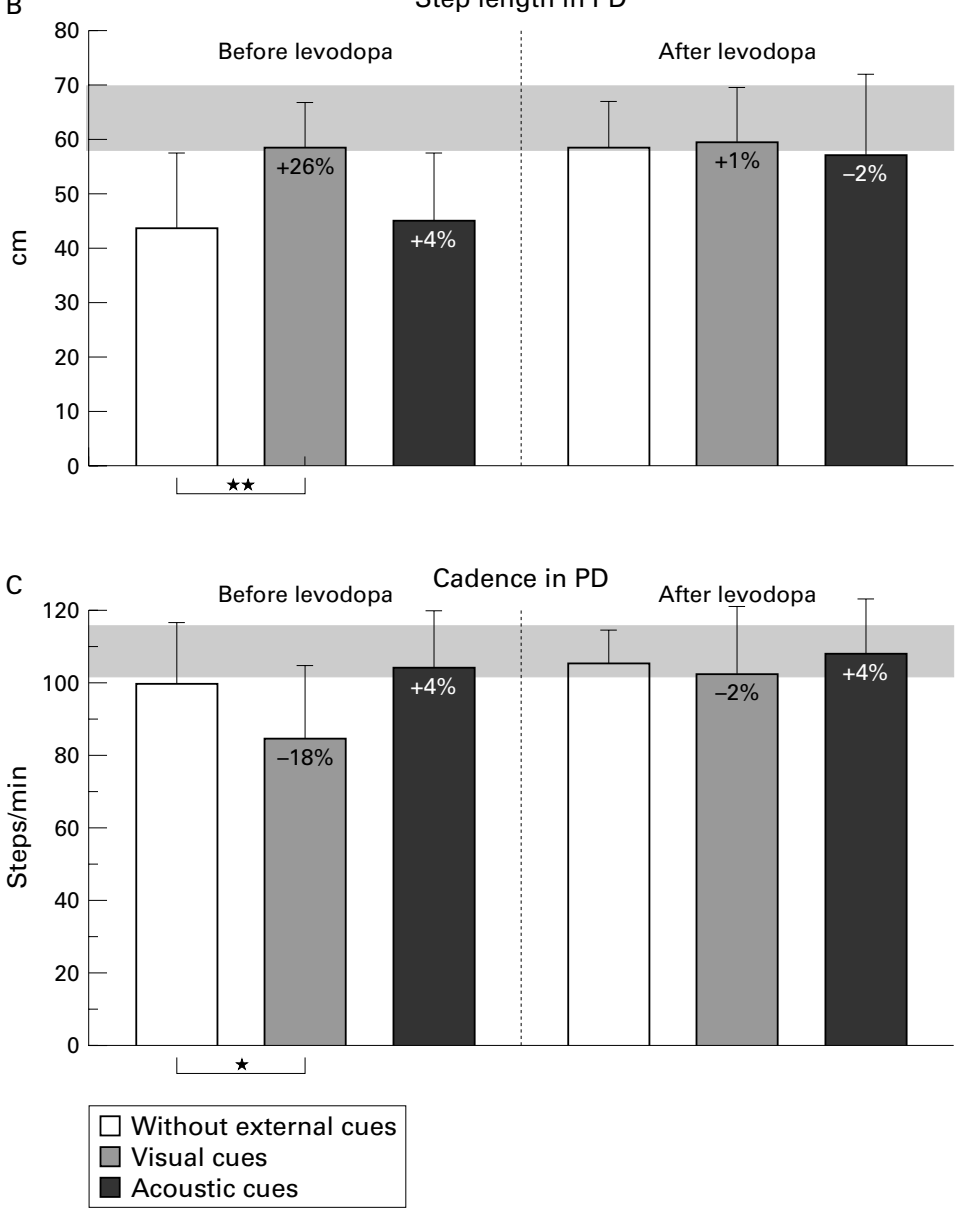

Figure 5 Effects of visual cues (stripes on the floor, displaying a normal step length) and acoustic cues (beat of a metronome corresponding to a normal cadence) on $(A)$ the gait velocity, (B) step length, and (C) cadence in patients with Parkinson's disease (PD) before (left bars) and after levodopa administration (right bars). Depicted are means (SD) of the gait parameters. The horizontal grey bar depicts the $95 \%$ CI of the mean values of each gait variable in the control group. Note the diminished cadence in normal pressure hydrocephalus (NPH), when visual cues were applied to the patients before levodopa treatment. Significant differences between the conditions with and without cues are indicated: ${ }^{*}<<0.05 ;{ }^{\star *} p<0.01 ;{ }^{\star *} p<0.001$ (before and after CSF tapping)

\section{Discussion}

The present study has shown new features of the gait disorder in normal pressure hydrocephalus and Parkinson's disease. Both share a hypokinetic gait due to a decreased stride length, but only patients with normal pressure hydrocephalus exhibit gait variables related to abnormal balance indicating a disturbance of the dynamic equilibrium differing from the one of Parkinson's disease. Appropriate therapeutic interventions improved the gait velocity in both conditions but left the disturbance of dynamic equilibrium in normal pressure hydrocephalus unaffected. As expected, external visual and acoustic cues improved the gait of patients with Parkinson's disease but a definite improvement was lacking in those with normal pressure hydrocephalus. These findings can act as guides to the clinical differential diagnosis of normal pressure hydrocephalus and Parkinson's disease and indicate differences in the underlying pathophysiology.

GAIT PATTERN IN PARKINSON'S DISEASE

The gait pattern in Parkinson's disease is characteristically altered, and has been well described in previous studies. ${ }^{12} 1416203233$ The trunk is bent forward, the joint movements are generally reduced, and the hip and knee joint are slightly flexed throughout the gait cycle. Arm swing is characteristically reduced. The steps are short and variable, whereas the cadence is mostly normal, except for later stages with severe akinesia. Nevertheless, frequency modulation of the cadence is limited. ${ }^{34}$ The step width and foot rotation angles are normal but invariable. ${ }^{33}$ More steps are needed to turn or to change the direction. In later stages of the disease the foot to floor clearance is reduced and the patient shuffles over the ground. Freezing and festination may occur.

The present study has clarified previous contradictory results and added some new findings concerning the features of gait in Parkinson's disease. After levodopa treatment the stride length increased whereas the variability from stride to stride decreased, confirming the results of a recent study ${ }^{35}$ and contradicting a study in which the variability remained uninfluenced. ${ }^{17}$ Temporal gait variables (the phases of the walking cycle) have been described to be levodopa resistant, assumed to be due either due to a disturbed coordination between postural control and locomotion, or the involvement of transmitter deficiencies other than dopamine. ${ }^{17}$ The results of the present study do not confirm these results, as an increase in the gait velocity must be followed by a change in the phases of the walking cycle (fig $3 \mathrm{~B}$ ).

It is common clinical experience that patients with Parkinson's disease respond excellently to visual and acoustic cues to overcome akinesia, which has been confirmed quantitatively. ${ }^{20-22} 36{ }^{37}$ Correspondingly, it has been shown that patients with Parkinson's disease rely more than healthy subjects on visual input. ${ }^{34}$ The results of the present paper (study 3 ) confirmed the responsiveness to both visual and acoustic cues, even when the patients were off levodopa. Interestingly, the cadence was diminished when the patients were instructed to step on the stripes placed on the floor, and further, this peculiarity was abolished after levodopa application. We interpreted this finding as an expression of disturbed motor programming, which is improved by levodopa. 
GAIT PATTERN IN NORMAL PRESSURE HYDROCEPHALUS

Compared with controls, we found the hypokinetic gait in normal pressure hydrocephalus to be characterised by the triad of reduced stride length, reduced step height, and a typical disturbance of the dynamic equilibrium. The stride length was markedly decreased, albeit symmetric and varied considerably from step to step. "Freezing" occurred in $30 \%$ of our patients. The foot to floor clearance was markedly reduced. The step width was enlarged and the feet were rotated outwards. The normal variability of step width and foot angles was decreased, leading to an insufficient compensation of body sway, as it is of particular importance during obstacle avoidance. These results suggest that a disturbed "dynamic equilibrium" during gait is the striking characteristic of the gait pattern in normal pressure hydrocephalus.

The first key feature of gait in normal pressure hydrocephalus is the reduction of stride length. ${ }^{381026}$ The changes of temporal gait variables has been controversially discussed in previous studies. One study found no differences in the phases of the walking cycle in patients with normal pressure hydrocephalus, ${ }^{38}$ but another group found a prolonged stance phase, whereas the swing phase duration was shortened. ${ }^{10}$ Our results confirmed the second finding. Compared with patients with Parkinson's disease lower values of the gait velocity, stride length, and the kinematics of gait in our sample might indicate that patients with normal pressure hydrocephalus were in a more severe stage of the disease. Although we tried to include patients in both groups with equal severity of disease this assumption cannot be excluded, as it is a common experience that patients with normal pressure hydrocephalus are in a moderate or severe stage of the disease, when admitted to hospital. However, we could show that balance related gait variables which most powerfully distinguish between normal pressure hydrocephalus and Parkinson's disease were not velocity dependent.

Surprisingly, the second characteristic, equilibrium related gait variables, have never been measured except in one study, in which the dynamic base was not found to be significantly enlarged. ${ }^{10}$ We found the broad based gait to be one of the most distinct abnormalities in our patients. Either the feet were rotated outward or the step width was enlarged (fig 2) and we interpreted this phenomenon as a protective strategy to stabilise locomotion. The third key feature was the diminished step height. We assume the reduced step height to be either a result of the rigidity of the lower limb muscles, or a further adaptation to improve stability during gait, such as someone walking on a slippery surface. Clinical observation of the patient's gait disclosed that associated movements such as trunk movements and arm swing were relatively preserved. During gait the trunk was held erect in most patients.

After CSF tapping in normal pressure hydrocephalus some gait variables were influenced (gait velocity, stride length), whereas others remained unaffected (cadence, balance related gait variables). Most striking, the gait velocity increased by about $21 \%$, due to an enlargement of the stride lengths, which were also more regular after tapping.

The responsiveness of locomotion in normal pressure hydrocephalus to external cues has never been investigated. Our results indicate that visual and acoustic cues improved the gait disturbance only slightly, both before and even after CSF tapping. Although external cues improved gait velocity, stride length, and cadence to a similar percentage as in Parkinson's disease, the resulting values were still far from the normal range. It is interesting to note that the cadence slowed down when the patients were instructed to step on the stripes, as in Parkinson's disease. We interpret this as an expression of an impaired motor programming, which means that different motor commands cannot be executed in parallel. This is a well known phenomenon in gait of patients with degenerative brain diseases such as Alzheimer's disease. ${ }^{39}$

Taken together, these findings stimulate speculations about the pathophysiology of gait disorder in normal pressure hydrocephalus. Although it has been traditionally thought that the gait disturbance might be due to alterations of the basal ganglia loop, ${ }^{23}$ the differences found in the present study between normal pressure hydrocephalus and Parkinson's disease are hard to reconcile with such a concept, as the two different physiological systemsbalance regulation and motor programmingare involved in the clinical picture of normal pressure hydrocephalus.

Firstly, the disturbance of balance in normal pressure hydrocephalus cannot be explained by basal ganglia defects as we found no similarities in balance regulation between Parkinson's disease and normal pressure hydrocephalus. Furthermore, the disturbed dynamic equilibrium in normal pressure hydrocephalus remained unaffected by the tapping procedure despite an increase in step length, which is a key finding. This indicates again that the subsystems for motor programming and balance must be involved differently.

Secondly, the fact that external cues are not helpful in improving gait in normal pressure hydrocephalus raises the hypothesis that a lack of internal cues from the basal ganglia to drive the supplementary motor area and motor cortex via the ventrolateral thalamus ${ }^{23}$ may play a minor part in the hypokinetic gait disorder in normal pressure hydrocephalus. Hypokinesia, freezing, and balance disturbance are often seen after frontal cortex lesions. Such gait disorders due to structural lesions of the frontal lobes and their connections have also been called "frontal dysequilibrium". ${ }^{40}$ As the basal ganglia loop is projecting to the frontal cortex this may explain some of the symptomatic overlap of hypokinesia in Parkinson's disease and normal pressure hydrocephalus. Balance is not exclusively regulated at the supraspinal level. It may also be abnormal after disturbances of corticobulbar fibres and subsequent disturbance of brainstem related posture and balance systems. Thus primary frontal damage, 
lesion of frontocerebellar pathways, or lesioned corticocortical fibres projecting to the frontal cortex are more likely to cause the condition.

BALANCE RELATED GAIT VARIABLES AS THE KEY FOR THE DIFFERENTIAL DIAGNOSIS OF NORMAL PRESSURE HYDROCEPHALUS AND PARKINSON'S DISEASE

The differential diagnosis of normal pressure hydrocephalus and Parkinson's disease is an everyday clinical problem. Most clinicians base the diagnosis of normal pressure hydrocephalus on the broad based gait and mostly preserved upper limb movements during gait, as well as urinary incontinence and signs of dementia. The complete triad of normal pressure hydrocephalus, however is exceptional and the earlier the diagnosis, the less clear are the symptoms. Conversely, Parkinson's disease is usually diagnosed if the symptoms have a unilateral accentuation. Imaging (CT or MRI) or appropriate therapeutic interventions are mostly the next step. We showed that not only enlarged step width but especially increased foot angles are almost never seen in Parkinson's disease whereas in normal pressure hydrocephalus a widened step width or larger foot angles are commonly present. This feature can be assessed clinically in a given patient. Secondly, visual and acoustic cues in our sample of patients with normal pressure hydrocephalus did not improve locomotion qualitatively. Furthermore, it is of special interest that after a spinal tap gait velocity is the most critical variable, even when assessed clinically, but the step width and foot angle do not respond. Whenever Parkinson's disease is suspected, a small based gait, favourably responsive to levodopa seems to be the best predictor according to our analysis. For a proper estimation of the clinical value of these variables a prospective clinical study is mandatory.

We kindly thank all patients and the subjects, who gave their time so generously and participated in the study. We acknowledge the work of Dr Johannes Pohl who prepared the statistical analysis and the support of Dr Stefan Palmie, who evaluated the MRI carefully. We thank Sibylle van der Horst, Andrea Dembinsky, and Matthias Kriest for help with evaluating the gait measurements. This work was supported by a grant Germany (BMBF FK: 01KO9511).

1 Sudarsky L. Clinical approach to gait disorders of aging: an overview. Philadelphia: Lippincott-Raven, 1997.

2 Thompson PD, Marsden CD. Gait disorder of subcortical arteriosclerotic encephalopathy: Binswanger's disease. Mov Disord 1987;2:1-8.

3 Tanaka A, Okuzumi H, Kobayashi I, et al. Gait disturbance of patients with vascular and Alzheimer-type dementias. Percept Mot Skills 1995;80:735-8.

4 Ebersbach G, Sojer M, Valldeoriola F, et al. Comparative analysis of gait in Parkinson's disease, cerebellar ataxia and subcortical arteriosclerotic encephalopathy. Brain 1999; 122:1349-55.

5 Adams RD, Fisher CM, Hakin S, et al. Symptomatic occult hydrcepahlus with "normal" cerebrospinal fluid pressure. N Engl f Med 1965;273:117-26.

6 Fisher CM. Hydrocephalus as a cause of disturbances of gait in the elderly. Neurology 1982;32:1358-63.

7 Graff-Radford NR, Godersky JC. Normal-pressure hydrocephalus. Onset of gait abnormality before dementia predicts good surgical outcome. Arch Neurol 1986;43:940-2.

8 Malm J, Kristensen B, Fagerlund M, et al. Cerebrospinal fluid shunt dynamics in patients with idiopathic adul hydrocephalus syndrome. $\mathcal{F}$ Neurol Neurosurg Psychiatry 1995;58:715-23.

9 Stolze H, Drucke H, Kuhtz-Buschbeck JP, et al. Gait analysis in normal pressure hydrocephalus: which parameters
respond to the CSF-tap test? Electroencephalogr Clin Neurophysiol 1998;106(suppl 1001):PS7-1.
10 Sudarsky L, Simon S. Gait disorder in late-life hydrocephalus. Arch Neurol 1987;44:263-7.

11 Krauss JK, Droste DW, Vach W, et al. Cerebrospinal fluid shunting in idiopathic normal-pressure hydrocephalus of the elderly: effect of periventricular and deep white matter lesions. Neurosurgery 1996;39:292-300.

12 Knutsson E. An analysis of Parkinsonian gait. Brain 1972;95:475-86.

13 Knutsson E, Martensson A. Quantitative effects of L-dopa on different types of movements and muscle tone in Parkinsonian patients. Scand F Rehabil Med 1971;3:12130.

14 Murray MP, Sepic SB, Gardner GM, et al. Walking patterns of men with parkinsonism. Am F Phys Med 1978;57:27894.

15 Stern GM, Franklyn SE, Imms FJ, et al. Quantitative assessments of gait and mobility in Parkinson's disease. $\mathcal{f}$ Neural Transm Suppl 1983;19:201-14.

16 Blin O, Ferrandez AM, Serratrice G. Quantitative analysis of gait in Parkinson patients: increased variability of stride of gait in Parkinson patients: incre
length. $\mathcal{F}$ Neurol Sci 1990;98:91-7.

17 Blin O, Ferrandez AM, Pailhous J, et al. Dopa-sensitive and dopa-resistant gait parameters in Parkinson's disease. $\mathcal{F}$ Neurol Sci 1991;103:51-4.

18 Bowes SG, Clark PK, Leeman AL, et al. Determinants of gait in the elderly parkinsonian on maintenance levodopa/ carbidopa therapy. Br f Clin Pharmacol 1990;30:13-24.

19 Brooks DJ, Salmon EP, Mathias CJ, et al. The relationship between locomotor disability, autonomic dysfunction, and the integrity of the striatal dopaminergic system in patients with multiple system atrophy, pure autonomic failure, and Parkinson's disease, studied with PET. Brain 1990;113: 1539-52.

20 Morris ME, Iansek R, Matyas TA, et al. The pathogenesis of gait hypokinesia in Parkinson's disease. Brain 1994;117: gait hypol.

21 Morris ME, Iansek R, Matyas TA, et al. Stride length regulation in Parkinson's disease. Normalization strategies and underlying mechanisms. Brain 1996;119:551-68.

22 Morris M, Iansek R, Matyas T, et al. Abnormalities in the stride length-cadence relation in parkinsonian gait. Mov Disord 1998;13:61-9.

23 Curran T, Lang AE. Parkinsonian syndromes associated with hydrocephalus: case reports, a review of the literature, and pathophysiological hypotheses. Mov Disord 1994;9: 508-20.

24 Hakim S, Adams RD. The special clinical problem of symptomatic hydrocephalus with normal cerebrospinal fluid pressure Observations on cerebrospinal fluid hydrodynamics. $\mathcal{F}$ Neurol Sci 1965;2:307-27.

25 Jack CR Jr, Mokri B, Laws ER Jr, et al. MR findings in normal-pressure hydrocephalus: significance and comparison with other forms of dementia. F Comput Assist Tomogr 1987;11:923-31.

26 Soelberg Sorensen P, Jansen EC, Gjerris F. Motor disturbances in normal-pressure hydrocephalus. Specia reference to stance and gait. Arch Neurol 1986;43:34-8.

27 Vanneste J, Augustijn P, Tan WF, et al. Shunting normal pressure hydrocephalus: the predictive value of combined clinical and CT data. $\mathcal{F}$ Neurol Neurosurg Psychiatry 1993;56:251-6.

28 Hughes AJ, Daniel SE, Kilford L, et al. Accuracy of clinical diagnosis of idiopathic Parkinson's disease: a clinicopathological study of 100 cases [comments]. $\mathcal{F}$ Neurol Neurosurg Psychiatry 1992;55:181-4.

29 Stolze H, Kuhtz-Buschbeck JP, Mondwurf C, et al. Retest reliability of spatiotemporal gait parameters in children and adults. Gait Posture 1998;7:125-30.

30 Brinckmann P. The angle of gait (author's transl). Zeitschrift für Orthopàdie 1981;119:445-8.

31 Stolze $\mathrm{H}$, Kuhtz-Buschbeck JP, Mondwurf C, et al. Gait analysis during treadmill and overground locomotion in children and adults. Electroencephalogr Clin Neurophysiol children and adults

32 Ueno E, Yanagisawa N, Takami $M$. Gait disorders in parkinsonism. A study with floor reaction forces and EMG. Adv Neurol 1993;60:414-8.

33 Vieregge P, Stolze H, Klein C, et al. Gait quantitation in Parkinson's disease: locomotor disability and correlation to clinical rating scales. F Neural Transm 1997;104:237-48.

34 Dietz V, Zijlstra W, Prokop T, et al. Leg muscle activation during gait in Parkinson's disease: adaptation and interlimb coordination. Electroencephalogr Clin Neurophysiol 1995;97: 408-15.

35 MacKay-Lyons M. Variability in spatiotemporal gait characteristics over the course of the L-dopa cycle in people with advanced Parkinson disease [published erratum appears in Phys Ther 1999 Jan;79(1):96]. Phys Ther 1998;78:1083-94.

36 Morris ME, Matyas TA, Iansek R, et al. Temporal stability Morris ME, Matyas TA, Iansek R, et al. Temporal stability
of gait in Parkinson's disease. Phys Ther 1996;76:763-80.

37 Azulay JP, Van Den Brand C, Mestre D, et al. Automatic motion analysis of gait in patients with Parkinson disease: effects of levodopa and visual stimulations. Rev Neurol (Paris) 1996;152:128-34

38 Knutsson E, Lying-Tunell U. Gait apraxia in normalpressure hydrocephalus: patterns of movement and muscle activation. Neurology 1985;35:155-60.

39 Camicioli R, Howieson D, Lehman S, et al. Talking while walking: the effect of a dual task in aging and Alzheimer's disease. Neurology 1997;48:955-8.

40 Nutt JG, Marsden CD, Thompson PD. Human walking and higher-level gait disorders, particularly in the elderly [comments]. Neurology 1993;43:268-79. 